\title{
REVISIONES
}

\section{Educación Física y transformación social: implicaciones desde una epistemología posmoderna*}

\author{
Physical Education and social transformation: implications from a post- \\ modern epistemology
}

Educação Física e transformação social: implicações a partir de uma epistemologia pós-moderna

\author{
Álvaro Sicilia Camacho \\ Universidad de Almería, Facultad de Ciencias de la Educación. Ctra de Sacramento, s/n, 04120, La Cañada \\ de San Urbano, Almería, España. Telf.: +34 9500153 94. Correo electrónico: asicilia@ual.es
}

\section{RESUMEN}

Este artículo parte de la premisa de que conocimiento y realidad son conceptos clave en la transformación social, dado que, dependiendo de cómo entendamos la realidad y la forma de acercarnos a ella, justificaremos ciertas prácticas frente a otras. Asumiendo esta premisa, el artículo presenta dos concepciones epistemológicas que han guiado la tradición occidental y que siguen vigentes a la hora de informar nuestras prácticas educativas. No obstante, estos sistemas de creencias se presentan problemáticos tanto en sus aspectos epistemológicos como ideológicos, por lo que el artículo delinea también una concepción posmoderna del conocimiento que pueda competir con las visiones tradicionales. Finalmente, cinco implicaciones pedagógicas derivadas de la visión posmoderna son presentadas con el objeto de permitir nuevas vías para la transformación de la educación, la educación física y la sociedad.

Palabras clave: posmodernismo, educación, conocimiento, currículo, pedagogía crítica.

\begin{abstract}
In this paper it is assumed the premise that knowledge and reality are both key concepts for social transformation due to the fact that any educational practice will be defended depending on how we understand and how we access to reality. From this premise, this paper shows two epistemological conceptions guiding the occidental tradition and informing our educational practices. Nevertheless, those belief systems are both epistemologically and ideologically problematic, so this paper also outlines a postmodern conception of knowledge which can compete with traditional views. Finally, five pedagogical implications from the postmodern view are showed in order to allow new ways of transformation for education, physical education and society.
\end{abstract}

Key words: postmodernism, education, knowledge, curriculum, critical pedagogy.

\section{RESUMO}

Parte-se da premissa de que conhecimento e realidade são conceitos-chave na transformação social, já que a forma de compreender a realidade e a forma de situar-se nela justificarão certas práticas no lugar de outras. Assume-se esta premissa, apresentam-se duas concepções epistemológicas que têm guiado a tradição ocidental e que ainda estão em vigor quando informam-se sobre práticas educativas. No entanto, estes sistemas de crenças se apresentam problemáticos tanto em seus aspectos epistemológicos como ideológicos, de modo que descreve-se uma concepção pós-moderna

* Este artículo fue solicitado por la Revista Estudios Pedagógicos en diciembre del 2011 en el contexto del proyecto de Investigación FONDECYT (Fondo de Investigación Científica y Tecnológica) No 11110016 , titulado "Educación Física y su función de transformación de las desigualdades sociales: profesorado del área y documentación ministerial”. El artículo fue aceptado en junio de 2012. 
de conhecimento que pode competir com as versões tradicionais. Finalmente, apresentam-se cinco implicações pedagógicas decorrentes da visão pós-moderna, a fim de permitir novas formas de transformação da educação, Educação Física e sociedade.

Palavras chave: pós-modernismo, educação, conhecimento, currículo, pedagogia crítica.

\section{INTRODUCCIÓN}

Todos los que trabajamos en el campo de la educación física (EF) estamos conformados con determinadas realidades educativas y, en ocasiones, nos enfrentamos con el reto profesional de querer intervenir sobre esas realidades para intentar producir cambios. Sin embargo, para actuar deliberadamente sobre la realidad es necesario disponer de un conocimiento de esa realidad que intentamos cambiar. Conocimiento y realidad son aquí conceptos clave, pues, dependiendo de cómo entendamos la realidad y la forma de acercarnos a ella, justificaremos ciertas prácticas en EF frente a otras e intentaremos influir en ellas de modos diferentes. De esta forma, diferentes conceptos sobre lo que es el conocimiento y cómo nos relacionamos con el mundo nos llevarán a diferentes formas de entender la EF y sus prácticas. Por ejemplo, si creemos que el conocimiento sobre el mundo es una cuestión fundamentalmente intelectual, entonces el cuerpo estará al servicio del intelecto, considerado como una cárcel de la mente. En esta visión, la EF suele centrar sus esfuerzos en mantener un cuerpo saludable y en forma, de tal modo que garantice un desarrollo apropiado de la psique. $\mathrm{Si}$, por el contrario, consideramos que la relación con el mundo es fundamentalmente corporal, pues el cuerpo es el elemento externo que primero toma contacto con el mundo y se posiciona dentro de él, entonces la práctica pedagógica en EF se basará en una indagación de las posibilidades corporales para relacionarnos con los demás y el mundo. De uno u otro modo, las creencias sobre el conocimiento nos informan, justifican y sostienen nuestras prácticas en EF.

Asumiendo esta cuestión, en este artículo presento de forma resumida la lógica de dos concepciones epistemológicas que han guiado la tradición occidental. En gran medida, estas concepciones siguen vigentes hoy día, e informan las prácticas educativas en las que participamos profesores y estudiantes. No obstante, estos sistemas de creencias son problemáticos tanto en su aspecto epistemológico como ideológico, por lo que el artículo delinea también una concepción posmoderna del conocimiento que pueda rivalizar con las visiones tradicionales. Cabe decir que, al presentar esta perspectiva, no es mi intención destruir las visiones tradicionales, sino sólo ofrecer una alternativa que puede ser considerada a la hora de plantear una transformación de la EF y la sociedad.

\section{LAS VISIONES TRADICIONALES DEL CONOCIMIENTO: LA VISIÓN REA- LISTA Y LA VISIÓN IDEALISTA}

Aunque existen muchas formas de presentar nuestras tradiciones históricas, puede ser útil en este artículo señalar dos orientaciones ya tradicionales en la forma de conocer: una visión realista, centrada en el mundo, frente a una visión idealista, centrada en la mente. La tradición realista hunde sus raíces en el pensamiento aristotélico, recorriendo toda la filosofía empirista del conocimiento, es decir, desde Thomas Hoobes y John Locke hasta el positivismo lógico. La tradición idealista se nutre más en las ideas de 
Platón, pero se une a la tradición racionalista, desde René Descartes e Immanuel Kant hasta la inteligencia artificial. Ambas orientaciones comparten un dualismo mente/mundo en el que la existencia de un mundo externo, una realidad material, es enfrentada a la existencia de un mundo psicológico (cognitivo, subjetivo, simbólico).

Como se muestra en la Figura 1, desde la visión realista se aspira a que el conocimiento refleje la realidad tal y como es. En este sentido, se reconoce una posibilidad de acceso directo a la realidad. El conocimiento válido se adquiere cuando los estados internos del individuo reflejan o representan apropiadamente la realidad, sirviendo como un espejo del estado existente ahí fuera. Los pensadores característicos de una visión realista suelen dar un papel muy importante a la observación como medio de conocer, de tal modo que tienden a ver lo emocional y los valores personales como una amenaza a la neutralidad necesaria para captar apropiadamente el mundo tal y como es. Además, la visión realista probablemente enfatiza la importancia del conocimiento como posibilidad que tiene el sujeto de adaptarse o tener éxito dentro de un ambiente complejo. Así, se considera que la persona debe tener un mapa interno de la naturaleza, que refleje, tal y como es, lo que hay ahí fuera. En definitiva, para la visión realista el mundo es dado, es como es, y la persona posee un conocimiento real y válido cuando refleja el mundo apropiadamente.

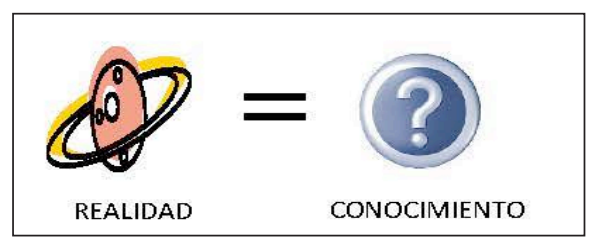

Figura 1. Visión realista del conocimiento

La visión idealista considera que no es posible un acceso directo a la realidad. El conocimiento es un producto de la percepción que el sujeto realiza de la realidad, es decir, de la interpretación que la persona hace del mundo (véase Figura 2). En este sentido, mientras la tradición realista trata la observación atenta del mundo como una clave para adquirir conocimiento, la idealista sitúa más el énfasis en la capacidad de interpretación racional del sujeto. Donde el educador realista probablemente se centraría en focalizar sobre los recursos del entorno necesarios para construir una representación apropiada, el educador idealista prestaría más atención a las capacidades intrínsecas de la persona para el desarrollo lógico o conceptual.



Figura 2. Visión idealista del conocimiento 
Como se sugirió, cada una de estas orientaciones del conocimiento sirve para justificar o racionalizar ciertas formas de prácticas en EF. Para un educador realista el conocimiento sobre la realidad está representado en las diferentes áreas curriculares. Desde esta visión el estudiante es percibido como una tabula rasa sobre la cual el proceso educativo debería inscribir las características esenciales del mundo. En concreto, esta perspectiva favorece la observación directa del estudiante o la experimentación del estudiante a través de situaciones prácticas. Los libros y la teoría son también aceptados por los realistas en la medida en que esto significa que el individuo puede adquirir más información que si sólo se limitara a una observación o experimentación directa con la realidad. De hecho, la suposición es que el conocimiento recogido en los libros de texto, o dictado por el profesorado en sus clases, es un conocimiento que ha sido constatado a través de la investigación y experimentación previa. La visión realista se muestra favorable a procesos de evaluación que determinen los niveles de conocimiento que el estudiante va adquiriendo. Así, para conocimientos como la anatomía del cuerpo o los procesos metabólicos del ejercicio, los recursos como preguntas de elección múltiple, test estandarizados y la normalización estadística pueden revelar en qué grado cada estudiante conoce la realidad, es decir, en qué grado un estudiante posee una representación apropiada, en cantidad y calidad, del mundo. Dada la importancia de conocimientos procedimentales en EF, el profesorado con una visión realista presta también mucho tiempo y dedicación a que el estudiante experimente prácticas motrices y deportivas. Diferentes test psicomotrices y pruebas deportivas serán utilizadas para determinar en qué grado de desarrollo psicomotor y deportivo se encuentra el alumnado.

Por el contrario, la visión idealista se centra más en el papel del estudiante para conocer la realidad. Un educador idealista se inclina por un currículo que enfatiza las capacidades racionales del individuo. En otras palabras, lo importante en el aprendizaje no sería la cantidad de información que uno adquiere, sino la manera en que uno delibera con esa información. Una EF guiada por una visión idealista se centraría en el objetivo de favorecer las capacidades del alumnado para pensar. Consiguientemente, esta visión guía maneras diferentes de actuar en clase. Procedimientos como la interrogación e indagación son fuertemente potenciados en el aula de cara a resaltar las habilidades cognitivas. De igual forma, la evaluación estaría al servicio de constatar el grado de comprensión y asimilación de aprendizajes, más que la memorización de los mismos. Así, para el ámbito de los aprendizajes conceptuales el desarrollo de trabajos de reflexión es preferido a los exámenes estandarizados (e.g. tipo test o preguntas cerradas). En la misma línea, para el ámbito del aprendizaje procedimental se enfatiza más el entendimiento y comprensión de la acción motora, más que su ejecución o repetición. Un ejemplo claro de las implicaciones pedagógicas que conlleva una u otra visión para la EF puede ser observado en el campo de la enseñanza deportiva. El modelo de enseñanza comprensiva, donde los gestos técnicos son adaptados y aprendidos a partir de situaciones reales y variadas de juego, se mostraría más en línea con una visión idealista del conocimiento. Por el contrario, un modelo basado en el aprendizaje repetitivo de la técnica, como paso previo al aprendizaje de situaciones tácticas, representaría una idea más o menos cercana de lo que sería la visión realista. 


\section{LA VISIÓN POSMODERNA DEL CONOCIMIENTO}

Aunque la práctica pedagógica actual en educación, y en EF, es fruto de la racionalidad de las visiones anteriormente descritas, estas tradiciones se muestran claramente insuficientes para la acción y la transformación social. La invalidez de la visión realista e idealista para una educación física transformativa viene en parte explicada por la sobredimensionalidad que éstas han puesto en el papel del sujeto para conocer. Desde la visión posmoderna hay, sin embargo, un nuevo argumento que emerge. Tanto la tradición realista como la idealista sitúan el conocimiento como algo extraído de una posible realidad. Para la visión realista el conocimiento es la aprehensión directa de una realidad (Figura 1). Para la visión idealista, el conocimiento es más bien producto de la interpretación que hacemos de esa realidad (Figura 2). En ambos casos, es el individuo quien observa o piensa sobre la realidad, a quien se le requiere adquirir o formarse una idea de algo que hay ahí fuera. Es precisamente con este conocimiento con el que el individuo se supone que puede sobrevivir y guiarse dentro de un mundo complejo. La visión posmoderna, por el contrario, invierte la relación entre el la realidad y el sujeto. No existe primeramente una realidad a partir de la cual el sujeto pretende conocer. La relación es más bien a la inversa. Como se muestra en la Figura 3, la visión posmoderna considera que el sujeto, en interacción con sus iguales, produce la realidad, generando un conocimiento particular sobre esa realidad. Por lo tanto, si la metáfora usada para la visión realista e idealista sería respectivamente la de descubrir y la de interpretar, - en ambos casos una realidad que está ahí fuera-, para la visión posmoderna el construir realidades sería la metáfora más apropiada.



Figura 3. Visión posmoderna del conocimiento

Al poner el acento en la construcción de la realidad, la visión posmoderna quiere llamar la atención sobre los efectos del conocimiento en las vidas de las personas. En otras palabras, la visión posmoderna denuncia las visiones anteriores por el hecho de centrarse en el conocimiento y no tanto en sus efectos. Si nos limitamos a aceptar que el conocimiento es algo esencial para que una persona pueda actuar en el mundo y sobrevivir, pero que éste reside en las cabezas de las personas individualmente, estamos limitándonos a reconocer nuestra práctica cultural y, con ello, consideraremos el conocimiento como un producto que surge fuera de la misma producción social. Los efectos más evidentes de considerar el conocimiento como una cuestión neutra es que nuestro propio conocimiento queda acotado. Preguntas sobre qué grupos salen privilegiados o qué tradiciones son borradas o suprimidas se hacen inviables. El conocimiento refleja muchas verdades, 
de tal manera que la verdad impuesta al individuo no deja de ser fruto de un continuo conflicto entre verdades. Como describió Foucault (1988), cada grupo o sociedad tiene su régimen de verdad, es decir, su política por la que establece lo verdadero y lo falso. La verdad es unida en una relación circular con los sistemas de poder que la producen y la sostienen, y con los efectos de poder que se derivan de ella.

Gran parte del esfuerzo de la visión posmoderna ha estado centrada en restaurar el papel constructor del mundo y en reconocer como cultural mucho de lo que había sido declarado como dado y natural. Al hacer esto, la visión posmoderna aboga por reemplazar la asunción de una verdad, bien verificada por la naturaleza o por imperativos categóricos, por una verdad creada en comunidad y en el seno de un juego constante de relaciones de poder. En otras palabras, la visión posmoderna renuncia a las pretensiones de establecer una verdad independiente de nosotros. Como afirmaba Nietzsche (1996), la verdad sólo puede ser considera como algo trascendente a nosotros desde el momento mismo en que olvidamos que somos quienes precisamente la inventamos. En sus palabras:

Si alguien esconde una cosa detrás de un matorral, a continuación la busca en ese mismo sitio y, además, la encuentra, no hay mucho de qué vanagloriarse en esa búsqueda y ese descubrimiento; sin embargo, esto es lo que sucede con la búsqueda y descubrimiento de la 'verdad' dentro del recinto de la razón (Nietzsche, 1996: 28).

Por lo tanto, lo que la visión posmoderna viene a recordar es que la verdad depende de nosotros mismos y que no existe una instancia fuera de nosotros, sea ésta Dios, la ciencia, las leyes o la realidad, que la establece o la regula. Así, los criterios de verdad los establecemos nosotros y, en consecuencia, son contingentes y relativos a nuestras prácticas. Los criterios de verdad no nos trascienden, somos nosotros los que definimos qué es verdad y qué no. Sin embargo, no establecemos los criterios en función de nuestro capricho, sino en función de unas reglas de juego que han sido social e históricamente establecidas. Esto implica que el conocimiento no es un producto individual sino fruto de las relaciones de poder. La visión posmoderna, de este modo, reivindica que todas las proposiciones de lo real o lo bueno tienen sus orígenes en las relaciones, es decir, es un producto eminentemente humano que se desarrolla en sociedad, y no al margen de ella. El conocimiento sobre el mundo tiene un carácter generativo, se produce en un proceso continuo de la acción coordinada de las personas. Ahora bien, que la verdad del conocimiento dependa de nosotros mismos, no significa que no sea útil para el grupo que lo establece y lo usa.

La visión posmoderna abraza un relativismo por el que se abandona la idea tradicional de que el conocimiento y la verdad tienen unos fundamentos últimos. Es precisamente en el momento en que abandonamos la creencia en la objetividad, y sustituimos la verdad absoluta por una verdad relativa y útil para nuestras prácticas, cuando se hace irremediable la presencia de los valores. Si no hay verdades fuera de nosotros se hacen evidentes algunas preguntas: ¿quién dice lo que es verdad, bueno, justo? ¿a quién beneficia y perjudica estas definiciones? ¿Qué mecanismos llevan a su aceptación y reproducción?

Dado que la visión posmoderna invierte la relación entre el conocimiento y la realidad, han sido frecuentes las posiciones de incomprensión desde otras formas de pensamiento. En la obra "Municiones para disidentes. Realidad-Verdad-Política", Tomás Ibáñez (2001) presenta de una forma clara y sencilla argumentos a favor de un relativismo posmoderno, a la vez que sugiere algunas implicaciones para nuestras vidas. El autor contesta a tres de las descalificaciones típicas que suelen ser vertidas contra el relativismo posmoderno. 
En primer lugar, se suele tachar al relativista posmoderno por poner en duda la ciencia y las verdades que han permitido el progreso científico. Sin embargo, lo que la visión posmoderna denuncia es que pueda haber parcelas de conocimiento que se sitúen fuera de la investigación y la reflexión, lo que implica más bien una actitud mucho más científica. Por lo tanto, la visión posmoderna no niega la ciencia, sino precisamente la reconoce y se implica con ella. Investigar y cuestionar todo lo que nos rodea es una actitud más fiel con el ethos científico.

En segundo lugar, el posmoderno, dado su relativismo, es a veces tachado como una persona con la que no se puede argumentar. ¿Si todo es relativo como se puede mantener argumentos para el debate? Sin embargo, la visión posmoderna más bien sugiere lo contario, es decir, que lo que pone fin al diálogo es precisamente que una de las partes recurra a absolutos desde los que es imposible el debate. Si hay cosas que son así y siempre han sido así, entonces poco espacio queda para su cuestionamiento. Una visión posmoderna reacciona claramente ante este tipo de posiciones.

En tercer lugar, pueden encontrarse descalificaciones hacia el que mantiene posiciones posmodernas, al entender que ello implica negar criterios éticos que sustentan un mundo más justo y humano. Sin embargo, una visión posmoderna reclama con más fuerza la necesidad de llegar a compromisos éticos, precisamente porque lo que niega es que existan principios éticos universales que nos trasciendan. Si los criterios éticos son una cuestión que compete al grupo, el compromiso con ellos surge desde su propia definición, de tal forma que existe una necesidad constante de mejorarnos mediante la argumentación y el enfrentamiento con las diferentes posiciones que pueden mantener los demás.

\section{IMPLICACIONES PEDAGÓGICAS DE UNA VISIÓN POSMODERNA}

Hemos descrito varios problemas inherentes en las concepciones tradicionales del conocimiento y hemos delineado las ideas de una visión alternativa posmoderna. Lo que nos proponemos ahora es explorar las implicaciones que esta visión posmoderna presenta para la EF. Antes de hacerlo, dos advertencias me gustaría realizar. En primer lugar, en mi exposición no hay intención de abandonar las tradiciones de conocimiento y de la pedagogía que arrastramos y, por supuesto, de imponer una forma única de entender la visión posmoderna. La visión posmoderna, o alguna de sus posibles interpretaciones, no reclaman ser una mejor perspectiva, un mejor pilar sobre el que un mundo nuevo pueda surgir. Así, no tengo interés en reemplazar las visiones anteriores en nombre de una nueva verdad, unos principios éticos más valiosos, una política más justa, o cualquier otro criterio universal. Más bien, mi intención es simplemente contribuir a aumentar y expandir los recursos y posibilidades existentes en servicio de un mayor bienestar. En segundo lugar, no cabe una única pedagogía normativa dentro de la visión posmoderna. Todas las prácticas sociales, tal vez con variedad de eficacia, sirven para construir diferentes mundos de lo real y lo bueno. En el mismo sentido, todas las pedagogías implican modos diferentes de entender la educación. Por ello, todas las formas de pedagogía han contribuido o contribuyen a dar significado a lo que se ha entendido por educación. La cuestión clave aquí es ver si las implicaciones que pueden derivarse de una visión posmoderna nos permiten abrir nuevas vías de partida para la transformación de la EF y la sociedad. Permítame el lector delinear sólo algunas de estas posibilidades. 


\subsection{DESAFIAR LA VISIÓN INGENUA DEL CONOCIMIENTO Y DEL CURRÍCULO}

Consistente con las visiones tradicionales, las cuales consideran el conocimiento como algo acumulativo y universal, las instituciones educativas son construidas alrededor de lo que Freire (1978) llamó modelo bancario de la educación. En este modelo el papel del educador y educado están claramente delimitados y definidos. El profesor es quien educa, sabe, piensa, habla, disciplina, prescribe, escoge el contenido, actúa, representa a la autoridad y guía el proceso. Por el contrario el estudiante es el educado, quien no sabe, no piensa, pero es pensado, escucha dócilmente, es disciplinado, acata las prescripciones, obedece, se acomoda al contenido, se adapta al profesor y se convierte, consiguientemente, en mero objeto del proceso de enseñanza.

El modelo bancario obedece a una visión jerárquica del conocimiento. La verdad la poseen los expertos, de tal modo que la autoridad y el poder reside en aquellos que producen el conocimiento. En este modelo, el papel del profesor es ser un transmisor de la verdad desde sus fuentes de producción a sus puntos de consumo. Se supone que, dentro de cada campo, los científicos y los académicos son los encargados de generar las ideas que deben ser aceptadas. En otras palabras, se espera que aquéllos revelen o descubran la verdad que rige el mundo y que los profesores enseñen esas parcelas de verdad a los estudiantes. Así, a partir de las fuentes de producción, el conocimiento es parcelado y diseñado a través del currículo de las materias y diferentes etapas educativas. Una vez diseñado y parcelado, éste es empaquetado en unidades didácticas y otro material. A veces, antes de que el profesorado pueda hacer uso de estas ideas y conceptos, existe un grupo de administradores y burócratas en diferentes niveles del sistema educativo (e.g. administraciones educativas, centros escolares, departamentos), que seleccionan las diferentes unidades que van a ser ofrecidas al alumnado. En este modelo educativo, el profesorado es sólo una pieza del engranaje situada al final del proceso, como un instrumento que dispensa los nutrientes básicos que harán que el alumnado pueda ir adaptándose a este mundo. Por supuesto, del estudiante sólo se espera que en último lugar consuma los conocimientos que le son ofrecidos por el profesorado en sus clases.

Aunque el modelo bancario descrito por Paulo Freire (1978) ha sido ampliamente criticado en la literatura educativa (Apple, 1986, 1987, 1989; Bourdieu y Passeron, 1973; Bernstein, 1988; 1989, 1993), lo cierto es que sigue bastante vigente en un gran número de escuelas y universidades. Por ejemplo, Apple (1989) ha documentado con bastante precisión el proceso jerárquico a través del cual el contenido educativo es producido y pasado a los profesores a través de los libros de textos y materiales curriculares producidos por otros. Dentro del campo de la EF, numerosos teóricos y académicos han señalado que los programas de formación del profesorado siguen una visión tecnocrática y de rendimiento que, en líneas generales, recuerda también este modelo bancario (Fernández-Balboa, 1995, 1997; Kirk, 1990, 1993; McKay et al. 1990; Sicilia y Fernández-Balboa, 2006; Tinning, 1991). Con esta visión de la enseñanza, la formación del profesorado en EF pone el acento más en el cómo aprender a enseñar los contenidos y no tanto en el para qué o por qué deben ser transmitidos. Este modelo de currículo conlleva también una forma de pensar el cuerpo que contribuye a la formación de determinadas subjetividades y a la reproducción de una economía y un modelo de producción desigual (Barbero, 2005; Sicilia, 2009; Wright, 2000). 
Como ha destacado reiteradamente la teoría educativa (Apple, 1989; Giroux, 1990; Contreras, 1997; Tinning, 1991), un modelo bancario de enseñanza descalifica tanto al profesor como el estudiante. Por un lado, aunque pudiera pensarse que una enseñanza basada en un modelo jerárquico del conocimiento dota de autoridad al profesor, lo cierto es que tal cosa es una ilusión. En este modelo el profesor participa poco de las decisiones sobre la producción del conocimiento y el diseño del currículo. Un modelo bancario de enseñanza lleva a una currículo estandarizado, lo que termina por imponer una descualificacion del profesorado (Apple, 1989). Como han detallado Aronowitz y Giroux (1991), las reformas educativas mantienen en común el papel que asignan al profesorado, donde su rol suele quedar reducido a un mero trabajador. Al ser tratados como técnicos y encomendarles fundamentalmente la implementación de un currículo que ha sido planificado por otros, el profesorado pierde su capacidad para reflexionar sobre las cuestiones principales y desarrollar posibles soluciones (Giroux, 1990, Contreras, 1997). Por otro lado, un modelo jerárquico del conocimiento descalifica al estudiante, limitando su creatividad e innovación. Sin embargo, esta descualificación no es inofensiva, sino que arrastra, al menos, dos claras consecuencias que deberían ser indicadas. En primer lugar, la descualificación no ocurre igual en todos los grupos de estudiantes. Dado que el conocimiento profesional es generado en un segmento de la sociedad, atiende más a los interés de unos grupos que otros (e.g. blancos, clases media y altas, hombres, payos). De este modo, los discursos de la escuela son más significativos dentro de unos contextos que otros, y, como ha detallado la investigación ampliamente, los estudiantes que se enfrentan a estos discursos desde otros sectores de la sociedad (i.e. clases obreras, gitanos, mujeres, otras etnias) pueden encontrarlos más irrelevantes y remotos (Apple, 1986; Bernstein, 1988, 1989, 1993; Woods y Hammersley, 1995). En segundo lugar, la descualificación oculta el papel del cuerpo y sus variadas formas en el proceso de aprendizaje (Wright, 2000). No se trata de reconocer que el cuerpo pueda ser visto como representación discursiva y transmisor de mensajes y relaciones de poder, sino también, como expresan Evans, Davies y Rich (2009), que el cuerpo en sí actúa como un dispositivo pedagógico. Es decir, el cuerpo tiene su voz propia, funciona como un conductor que tiene en cada persona su propia sintáctica y gramática interna, que viene dado por la intersección de la biología y la cultura, y que regula (facilitando o constriñendo) los aprendizajes, incluyendo la manera en que los mensajes discursivos son leídos y reconocidos.

Dado los problemas inherentes a un conocimiento jerárquico nos queda preguntarnos qué soluciones pueden ser presentadas. La visión posmoderna añade una nueva dimensión a estas críticas. En concreto, ve todo reclamo al conocimiento como insertado dentro de comunidades particulares que construyen significado. Una visión posmoderna sugiere desacralizar el conocimiento profesional y presentar alternativas y acciones diferentes. Más que asumir que el conocimiento tradicional proporciona la mejor o la última palabra, debemos tomar conciencia que todo reclamo de conocimiento surge de tradiciones cultural e históricamente situadas. Esto no es negar su valor en absoluto, sino aceptar que tales valores son también contingentes.

De cara a la práctica educativa, la visión posmoderna conlleva a un compromiso por comunicar a los niños y niñas que lo que aprenden como verdad es lo que es mantenido como verdad por la sociedad en la que pertenecen. Así, una visión posmoderna no está reñida con transmitir convenciones establecidas en el grupo. Aprender lo que un grupo ha consensuado como temporalmente verdadero servirá al estudiante para integrarse y 
adaptarse en la sociedad en la que vive. Sin embargo, integrado con estas convenciones debería estar la evaluación y la crítica. Aprender el conocimiento que otros han producido históricamente, como una convención que funciona en mi grupo y en el momento actual, es un primer paso para poder en el futuro participar dentro de grupos que puedan cuestionar, revisar y, llegado el caso, proponer cambios de lo que debería ser considerado como válido y verdadero para mi grupo. Ser un profesor de EF significa pertenecer a una comunidad que se guía por reglas y principios más o menos establecidos y que han ido variando a lo largo de los años (Sicilia y Fernández-Balboa, 2006). No obstante, estos principios no siempre coinciden con nuestra visión de la EF. La necesaria existencia de posiciones alternativas y, a veces, contrapuestas, es lo que la visión posmoderna destaca como algo esencial en las mismas relaciones sociales. Lejos de ir en detrimento de la profesión, la visión posmoderna reclama el potencial que puede tener el conflicto como multiplicidad de voces. Lejos de lo que la visión tradicional nos ha hecho entender, el conflicto no es algo necesariamente malo. El conflicto lleva a que las personas puedan discutir sobre lo que no están de acuerdo, en lugar de asumir lo que se ha establecido como cierto y verdadero para todos. La posibilidad de ver diferentes opciones implica la activación de la persona al tener que decidir alguna de las posibles. Si nos presentan una opción como la única posible, la elección ya está tomada. En consecuencia, la posibilidad de ver diferentes opciones nos lleva a valorar y elegir de forma más consciente e intencional nuestras acciones, en lugar de actuar basado en la tradición o en la mera inercia. Esto no implica un medio más seguro de pensar y actuar, sino simplemente ser algo más dueños de nuestros aciertos o errores.

\subsection{POTENCIAR RELACIONES GENERADORAS DE CONOCIMIENTO}

Tanto desde una epistemología realista como idealista el sujeto es situado fuera del proceso de producción del conocimiento. De este modo, las visiones tradicionales, al situar el conocimiento bien fuera del sujeto o bien dentro de la mente del sujeto, favorecen una distinción entre el profesor y el estudiante. El profesor conoce y los estudiantes alcanzan la posición de objetos sobre los que operar, llenando sus mentes de contenidos o enseñándoles a usar la razón para saber interpretar la realidad que está ahí fuera. Desde una visión posmoderna, sin embargo, el individuo no es el poseedor de contenidos o racionalidades sino más bien participa en ellas. Las afirmaciones de lo que es la realidad y lo que conocemos de ella son resultado de las relaciones sociales que son intrínsecas a la convivencia. Así, una visión posmoderna sugiere que el desafío para la educación, y para la EF, es contribuir a potenciar relaciones generativas, es decir, relaciones desde las cuales los estudiantes emerjan con potencial para relacionarse y participar activamente en la producción del conocimiento que rige la sociedad. Por lo tanto, la visión posmoderna aboga por un cambio en el rol del estudiante. Un cambio que implica pasar de ser objeto de la educación a un sujeto dentro de las relaciones donde se produce la actividad educativa.

Al ver el conocimiento de esta forma, lo que la visión posmoderna está sugiriendo es convertir el proceso de enseñanza-aprendizaje en un diálogo, deliberar sobre las cuestiones que importan, su valor y su relevancia. Algunas propuestas pedagógicas basadas en el aprendizaje cooperativo o colaborativo tienen en cuenta la participación del alumnado en el proceso de enseñanza. Sin embargo, lo importante aquí no es que 
el estudiante coopere o colabore en actividades con otros, sino en ver cómo la relación puede enriquecer el proceso pedagógico. Más que una clase centrada en el alumnado se trata de ver cómo debe constituirse el proceso educativo si la relación debe ser lo primordial. La idea no es cambiar la EF para una mayor atención al individuo, sino una mayor atención a la relación.

Tal vez una de las prácticas educativas a destacar por su interés en la creación de relaciones generativas es el proyecto conocido como comunidades de aprendizaje (Elboj et al., 2002). Las comunidades de aprendizaje se basan en un proyecto educativo que pretende contribuir a la transformación social y la igualdad social a partir de la superación del fracaso escolar. En España sus antecedentes se sitúa en la década de los 70 del siglo pasado, a partir de la experiencia desarrollada en un centro de educación de adultos en Barcelona (Sánchez, 1999). A mitad de los 90 este proyecto fue extendiéndose progresivamente en diferentes niveles educativos y ha llegado a gran parte de la geografía española y algunos países sudamericanos. La base metodológica de las comunidades de aprendizaje se centra en el aprendizaje dialógico (Aubert et al., 2008), con clara inspiración de la teoría de Paulo Freire y Jürgen Habermas. Aunque, epistemológicamente, la visión posmoderna no comparte la idea de poder alcanzar verdades universales a través de las intersubjetividad, sí comparte el diálogo como medio de búsqueda de soluciones. Las comunidades de aprendizaje concretizan el aprendizaje dialógico a través del trabajo en grupos interactivos. El profesorado intenta trabajar con la diversidad del aula y, por ello, en lugar de sacar a determinados alumnos para atender sus necesidades fuera del grupo, facilita la entrada de otras personas adultas al aula (e.g. otro profesorado, familiares, voluntarios). Al actuar así, enriquecen las experiencias del aula y potencian los aprendizajes en la misma dinámica de la interacción social. Los grupos interactivos están siendo desarrollados en diferentes áreas curriculares, incluida la EF. Además, este modelo de trabajo está sirviendo para enfocar de forma diferente la inclusión de grupos desfavorecidos como población gitana o discapacitados.

\subsection{SITUAR LOS APRENDIZAJES EN LOS CONTEXTOS DE PRÁCTICA}

En conexión con la jerarquía del conocimiento y las prácticas unidireccionales descritas anteriormente, las visiones epistemológicas tradicionales han derivado un modelo de enseñanza que tiende a suprimir las condiciones contextuales de producción del conocimiento y las posibilidades pragmáticas que éste puede tener en cada caso. Dentro de las diferentes áreas curriculares, los conocimientos son desubicados de sus contextos de producción y consumidos en las aulas sin una clara conexión con los contextos de uso. El área de EF, a pesar de su carácter práctico y vivencial, no está exenta de esta descontextualización. Así, el conocimiento que se generó en torno al deporte del baloncesto, por ejemplo, sirve para unir una comunidad específica, representada hoy día bajo una estructura federativa que aglutina a técnicos, administradores y deportistas. Por lo tanto, ese conocimiento específico sirve para definir y dar valor a una práctica deportiva concreta y ayuda a generar resultados de importancia dentro de esa comunidad. Sin embargo, cuando las proposiciones de conocimiento son sacadas de su contexto y son puestas en el terreno de un patio de colegio, la función pragmática de estos discursos se pierde. Los discursos y prácticas pierden su relevancia y los estudiantes deben buscar el modo en que estos aprendizajes pueden ser útiles para sus vidas. Un estudiante deberá 
aprender los tipos de bote o las formas de entrar a canasta en situaciones abstractas. El significado del lenguaje y de las acciones para su vida es apenas atendido. En su lugar, la irrelevancia del aprendizaje se contrarresta aumentando la credibilidad de las técnicas y las reglas que le servirán para aprobar la materia. En otras palabras, los estudiantes terminarán ejecutando los gestos técnicos de acuerdo a los cánones establecidos para ese deporte y conociendo el reglamento que rige su funcionamiento, pero, en la mayoría de los casos, poca utilidad tendrá en la vida de cada estudiante.

Como en el caso del baloncesto, la mayoría de los conocimientos que llegan a la EF y a las escuelas conllevan una reproducción de los contextos donde ese conocimiento se ha generado. Además, dado que los conocimientos de los expertos son considerados como sacrosantos, es decir, el producto de nuestras mejores mentes, éstos tienden a transmitirse a través de la jerarquía con una perspectiva estrecha y reproductiva (Bernstein, 1993). El resultado es que los discursos y prácticas generadas en el vértice de la jerarquía no son fácilmente adaptados para que los estudiantes puedan usarlos de una forma apropiada en el exterior de la escuela y a lo largo de su vida. Al igual que los estudiantes no podrán usar fácilmente las reglas y gestos técnicos aprendidos en EF para sus actividades físicas y lúdicas en sus barrios y parques, tampoco podrán emplear muchos de los argots y tecnicismos aprendidos en la escuela dentro de su vida cultural, pues sus significados están adscritos a unos dominios específicos de uso académico.

Desde la visión posmoderna, las proposiciones de conocimiento obtienen su significado dentro de contextos particulares de uso y funcionan como un medio de coordinar la acción dentro de estos contextos. El lenguaje y las acciones adquieren su valor a partir de la forma en que es usado por la gente en contextos específicos. En este sentido, el desafío para el profesorado es crear procesos educativos donde, en lugar de depositar hechos y teorías, y reproducir acciones y gestos, se generen contextos en los que los discursos, acciones y prácticas de uso sean unidos. Esto significaría crear contextos en los que el aprendizaje pueda ser vinculado a las necesidades y objetivos prácticos que los estudiantes tienen dentro de sus comunidades y grupo.

En los últimos años, dentro de la $\mathrm{EF}$, han existido propuestas educativas que han intentado ir más allá de un aprendizaje descontextualizado. Por ejemplo, en el campo del aprendizaje deportivo, el modelo de educación deportiva de Siedentop y colaboradores (Siedentop, 1994, 1998, 2002) o el modelo comprensivo de Bunker y Thorpe (Bunker y Thorpe, 1982; Devís y Peiró, 1992; Griffin y Butler, 2005; Thorpe et al., 1986) han transcendido el mero aprendizaje de gestos técnicos, entendiendo la educación deportiva como un medio para formar personas competentes e ilustradas deportivamente. Competentes en el sentido de desarrollar habilidades y estrategias que permitan al estudiante participar de forma apropiada en juegos dentro de su contexto. Ilustrada deportivamente, en la medida en que pretende que las personas comprendan los fundamentos del juego, sus reglas y tradiciones, de tal forma que le permita modificarlas, adaptarlas y reinventarlas en función de sus necesidades y situación. No obstante, dado que en un mundo posmoderno las funciones de la EF se han visto ampliadas más allá que la enseñanza deportiva (e.g. promocionar actividades saludables, formar ciudadanos), otros modelos similares han surgido para cubrir finalidades diferentes. Así, el modelo de responsabilidad personal y social de Hellison y colaboradores (Hellison, 2003; Hellison et al., 2000; Hellsion y Martinek, 2006; Wright y Burton, 2008) o el aprendizaje cooperativo de Dyson y co- 
laboradores (Dyson, 2002, 2010; Velázquez, 2010) no centran tanto su atención en las acciones motrices y deportivas, como en el fomento de habilidades sociales y cívicas.

En cualquier caso, estas propuestas curriculares se enfrentan con la difícil tarea de producir un aprendizaje situado dentro de instituciones que poco reflejan el entorno en el que el alumnado vive. Es por ello que una visión posmoderna estaría de acuerdo en modificar el currículo establecido y su estructuración y clasificación a través de años y cursos. La idea de desarrollar determinados contenidos en cursos específicos de forma estandarizada hace que muchas veces las actividades y aprendizajes de cada curso estén unidos sólo a un contexto inmediato y práctico de uso. En estas situaciones, los aprendizajes son aplicables sólo dentro del ambiente artificial y delimitado del sistema educativo. La visión posmoderna estaría a favor de prácticas pedagógicas donde los estudiantes trabajaran con los profesores, y otros miembros de la comunidad, para primero decidir y luego desarrollar las cuestiones importantes y relevantes en las que merecería la pena implicarse.

Las implicaciones pedagógicas de una visión posmoderna no sugieren necesariamente que deban inventarse nuevas prácticas pedagógicas, sino basta con recuperar y repensar prácticas que en algún tiempo fueron planteadas como alternativas a la educación tradicional. La cuestión central aquí es evitar desarrollar pedagogías centradas en el alumnado, pero sin el alumnado. Es decir, aprendizajes que no son demandados de algún modo por los que deben estar interesados en ellos. Las propuestas pedagógicas del trabajo por proyectos pueden cubrir la demanda posmoderna de encontrar un aprendizaje más contextualizado. Esta forma de trabajo se adscribe, como muchas otras, al conjunto de propuestas que defienden un aprendizaje centrado en el alumnado. Sin embargo, la clave de su propuesta es que parte de un problema de la vida real. A través de un problema que conecta con la realidad del alumnado se plantea una estrategia educativa integral, que tiene como pilar un trabajo cooperativo e interdisciplinar. La motivación para aprender está asegurada en la medida en que las situaciones plantean retos y desafíos reales, surgidas de las necesidades de los propios estudiantes. Éstos muestran preocupaciones por la ecología, los conflictos raciales, la violencia en el deporte, el aborto, las drogas, el doping, la industria de la música rock, las demandas que imponen la moda y la presión para alcanzar determinados modelos de cuerpo. Todos ellos son problemas reales, a través de los cuales los estudiantes pueden interactuar con las personas de la sociedad comprometidos en estos ámbitos, coleccionar materiales relevantes, leer libros y artículos, discutir con otros y formular visiones que puedan llamar a la atención de padres, deportistas, policías, empresarios o gobernantes. Una visión posmoderna es contraria a aislar la recepción y producción del conocimiento. Los mejores aprendizajes se producen cuando se participa, se colabora y se actúa en la propia construcción del aprendizaje.

\subsection{GENERAR UNA PRÁCTICA PEDAGÓGICA AUTOREFLEXIVA}

Al igual que una perspectiva crítica, la visión posmoderna considera que la acción pedagógica y el currículo no son elementos neutros, de tal forma que el papel del educador debería ser analizado en relación con las estructuras económicas, sociales y políticas. La acción pedagógica requiere una reflexión y análisis constante del profesor y los estudiantes para problematizar las verdades fijas y estables del sistema. En este sentido, en las últimas décadas se han señalado conexiones entre la pedagogía crítica 
y una visión posmoderna (Fernández-Balboa, 1997; Sicilia y Fernández-Balboa, 2005). De hecho, Bain (1997) destaca la siguiente línea argumentativa que ha sido utilizada para unir la pedagogía crítica y la posmodernidad: 1) la escuela y la EF son fruto de la modernidad; 2) la modernidad ha fallado, de tal modo que las sociedades occidentales se están moviendo hacia un pensamiento posmoderno que desafía los principios básicos de la modernidad; 3) para superar los fallos de la modernidad y sobrevivir dentro de la posmodernidad la EF necesita cambiar; 4) consiguientemente, la redefinición de la EF en la época posmoderna necesita tener un énfasis en la crítica y en la emancipación.

No obstante, a pesar de las aportaciones que la pedagogía crítica ha podido tener para la EF, la visión posmoderna se muestra cauta con las posibilidades que cualquier propuesta pedagógica tiene para la transformación de la sociedad (Biesta, 1998; Ellsworth, 1989; Gore, 1993; Gur-Ze'ev, 1998). Por ejemplo, feministas como Lather (1998) o Gore (1993) han resaltado que las preinscripciones universales que formula la teoría pedagógica crítica han encontrado difícil aplicación dentro de la diversidad que supone las situaciones reales de las aulas. De hecho, algunos pedagogos, aún dando muestra de conocer ampliamente la teoría y el discurso de la pedagogía crítica, han relatado abiertamente las dificultades encontradas a la hora de poner en práctica los principios de la pedagogía crítica (Ellsworth, 1989; Gore, 1993, 2003, Hickey, 2001, 2005). En esta línea, no parece extraño que Tinning (2002) haya llegado a afirmar que una cosa es defender posiciones críticas en EF y otra llevarlas a la práctica.

Por otro lado, las visiones excesivamente paternalistas y universales de la pedagogía crítica han encontrado fuertes recelos desde posiciones fundamentalmente posmodernas. Por ejemplo, algunos autores han denunciado el lenguaje dicotómico y la superioridad moral que ha intentado imponer la pedagogía crítica, en su intento por desprestigiar otras formas pedagógicas e imponer sus postulados y prácticas (Ellsworth, 1989; Ennis, 1997; O’Sullivan et al., 1992). Al actuar así, la pedagogía crítica se expone a caer en una fuerte contradicción, pues dentro de su lenguaje de liberación se materializan prácticas de imposición y represión. En palabras de Bain (1997), al crear un sistema de categorías dicotómico (e.g. nosotros vs ellos; oprimidos vs opresores; currículo de rendimiento vs currículo participativo), la pedagogía crítica, irónicamente, reproduce el pensamiento de la modernidad.

Así, aunque la visión posmoderna coincide con perspectivas críticas de la educación en la necesidad de problematizar el conocimiento dado, la visión posmoderna es consciente que este postulado debe aplicarse incluso al conocimiento del que uno participa. En otras palabras, la visión posmoderna entiende que no hay conocimiento fuera de nosotros y, por lo tanto, no hay propuesta pedagógica que esté libre de valores e ideologías, es decir, que no represente una forma particular de ver la educación y el mundo. La visión posmoderna se alinea con una visión crítica de la enseñanza, pero sus propuestas aspiran siempre a ser modestas (Tinning, 2002). Como han destacado Sicilia y Fernández-Balboa (2009), esto exigiría un doble desafío para la perspectiva crítica de la EF. Por un lado, necesita mantener un fuerte compromiso con el proyecto de igualdad y justicia que representa pero, por otro, debe evitar caer en la presunción de tener la mejor, o incluso la única, solución pedagógica para la transformación social desde la EF.

Atender a este doble desafío requiere una continua tensión entre presentar las ideas propias y permitir que éstas compitan con otras posibles maneras de pensar. Esto no significa que uno no pueda defender sus posiciones o pensar que sus acciones pedagógicas 
puedan tener un efecto de transformación en los estudiantes y en los contextos de relación en los que participan. Sin embargo, una visión posmoderna recuerda el riesgo que tenemos de sustituir en nuestras clases un régimen de verdad por otro. Nos advierte que el conocimiento que enseñamos es siempre una de las muchas posibilidades que existen. En línea con ello, asume el análisis de diferentes realidades y subjetividades, de tal forma que los estudiantes puedan ser conscientes de la existencia de diferentes formas y regímenes de verdad. La idea es generar en el alumnado formas alternativas de relacionar los contenidos que aprenden con su propia vida, evitando, en todo caso, elegir por ellos. En resumen, como señaló Ortega y Gasset (2002) un profesor debería hacer dudar a sus estudiantes incluso de lo que uno enseña. Esto pone en alerta a los estudiantes ante el intento de suprimir sus voces o decidir el profesor por ellos.

\subsection{ROMPER LA BALCANIZACIÓN PROFESIONAL}

Durante el periodo de la modernidad, el conocimiento válido por el que debe ser regido nuestro mundo ha estado principalmente representado por la ciencia. Sin embargo, en el proceso de su creación y desarrollo ha existido una delimitación continúa en campos de conocimiento. Esto ha generado lo que, desde la economía de la educación, se ha conocido como un proceso de cualificación específica y, a la vez, descualificación general. En otras palabras, hemos llegado a la contradicción de encontrarnos en una sociedad que, en su conjunto, posee conocimientos suficientes como para inventar y crear productos más sofisticados y complejos de los que nunca hemos sido capaz de generar $\mathrm{y}$, sin embargo, individualmente poseemos menos habilidades y conocimientos que los que una persona poseían hace varios siglos para sobrevivir (Janossy, 1980). Como ha destacado Giddens (1993), vivimos en sociedades donde la confianza en los sistemas de expertos se ha vuelto algo esencial para nuestras vidas. Generalmente, cada uno de nosotros participa en un número limitado de sistemas de expertos, sin embargo, depende en su vida diaria de otros muchos sistemas de los que apenas conoce su funcionamiento, pero en los que no le cabe más remedio que confiar plenamente (e.g. conducir el coche, viajar en avión, utilizar cualquier dispositivo tecnológico).

La escuela de masas, fruto de la racionalidad de la modernidad, se erige a partir de la división del trabajo y la parcelación del conocimiento. Los currículos se disponen de tal forma que los estudiantes son expuestos a una variedad de campos, y a una progresiva especialización a medida que ascienden por el nivel educativo. De hecho, la escuela, a través de su currículo, reproduce a su escala los campos de conocimiento de la ciencia (Goodson, 1988, 1995). Sin embargo, esta parcelación del conocimiento en el ámbito profesional tiene el peligro de implantar una cultura balcanizada. Como describe Hargreaves (1996), las culturas balcanizadas se caracterizan porque tienden a la formación de subgrupos con una permeabilidad reducida, de tal modo que el aprendizaje de los profesores se desarrolla sólo dentro de su propio grupo. Además, los subgrupos que configuran una cultura balcanizada tienden a ser estables y perdurar en el tiempo (e.g. profesor de educación física vs otras áreas; profesor de primaria vs profesor secundaria; pedagogo crítico vs pedagogo tradicional). Precisamente esta permanente dedicación al grupo hace que surja una vinculación especial a la subcomunidad donde cada profesional comparte gran parte de sus intereses y tiempo. A través de un proceso de socialización el profesional aprende a identificarse con el grupo al que pertenece y a ver el mundo y la 
educación desde un mismo punto de vista. Por ejemplo, la investigación en EF ha mostrado que el nivel educativo donde el profesorado desarrolla su trabajo influencia la creación de diferentes valores y creencias sobre el currículo (Behets y Vergauwen, 2004; Sicilia et al., 2006). Sin embargo, las identidades de los profesores no quedan sólo configuradas por las disciplinas que imparten o los niveles educativos a los que pertenecen, sino que dentro de cada disciplina también hay espacio para subgrupos o corrientes. Así, en EF podría hablarse de una corriente psicomotriz, deportiva, recreativa, de condición física y salud, entre otras (Hernández, 1996). Estos subgrupos no representan simplemente fuentes de identidad y significado, sino que son fuerzas que conllevan intereses. Por ello, en las culturas balcanizadas se hace difícil la colaboración para proyectos comunes, pues los grupos suelen observarse entre sí como posibles ganadores y perdedores. Los intereses de cada grupo son siempre antepuestos al debate y la búsqueda de proyectos comunes.

Ante las subculturas balcanizadas una visión posmoderna se muestra partidaria de encontrar formas de enseñanza y aprendizaje más liberalizadas de las demandas de las disciplinas. Esto sugiere que los profesores de EF deberían tener miras más amplias. Si la educación tiene que ver con animar la calidad y la eficacia del debate público, entonces los aprendizajes estandarizados en paquetes curriculares deben extenderse al desarrollo de habilidades para conformar problemas complejos y cambiantes dentro y fuera de la escuela. En otras palabras, cuestiones de interés público son materias de la educación y de la EF. Esto sirve para recordar que la EF, al igual que el resto de disciplinas escolares, deberían ser medios y no fines en sí mismas (Pascual, 1999, 2006).

\section{CONCLUSIONES}

En este artículo se ha presentado una visión posmoderna del conocimiento que denuncia los discursos disciplinarios en las práctica de la EF, y se alinea con un cambio de pedagogía que no esté tanto centrado en el sujeto como en las relaciones, una mayor conexión local del currículo del aprendizaje, una necesaria autorreflexión de la práctica pedagógica, una ruptura de las fronteras disciplinares y, en general, una mayor democracia para negociar qué importa en educación. Muchos de estos énfasis no son nuevos, ni en los discursos educativos, ni en la EF, y, en este sentido, una visión posmoderna apoya ciertas iniciativas ya existentes. Aunque una visión posmoderna problematiza otras formas de $\mathrm{EF}$, no hay nada en los argumentos presentados que sugiera un abandono completo de las prácticas educativas tradicionales o, incluso, un modo único y particular de afrontar los retos de la visión posmoderna en la educación y la educación física. Todas las prácticas construyen el mundo a su manera, desarrollan valores de cierto tipo y les presta ciertas características en lugar de otras. Así, dentro del mundo socialmente construido de la EF, las visiones son más expresiones de los compromisos y rechazos que sentimos hacia teorías y prácticas pedagógicas que compiten entre sí a la hora de definir qué es una buena EF. Es el lector y el profesional los que tienen la posibilidad de elegir entre las visiones que rivalizan entre sí, o incluso identificarse con varias de ellas. Por lo tanto, la visión posmoderna es una opción más que, en lugar de restringir, suma nuevas posibilidades. 


\section{AGRADECIMIENTOS}

Agradezco los comentarios realizados por Cornelio Aguila Soto, de la Universidad de Almería, sobre un borrador de este artículo.

\section{REFERENCIAS BIBLIOGRÁFICAS}

Apple, M. (1986). Ideología y currículo. Madrid: Akal.

Apple, M. (1987). Educación y Poder. Barcelona: Paidós/MEC.

Apple, M. (1989). Maestros y textos. Una economía política de las relaciones de clase y de sexo en educación. Barcelona: Paidós/MEC.

Aronowitz, S. y Giroux, H. (1991). Postmodern education. Minneapolis: University of Minnesota. Aubert, A.; Flecha, A.; García, C.; Flecha, R. y Racionero, S. (2008). Aprendizaje dialógico en la sociedad de la información. Barcelona: Hipatia.

Bain, L. (1997). Transformation in the postmodern era: A new game plan. En J. Fernández-Balboa (Ed.), Critical postmodernism in human movement, physical education, and sport (pp. 183195). Albany, NY: State University of New York Press.

Barbero, J. (2005). La escolarización del cuerpo: Reflexiones en torno a la levedad de los valores del capital "cuerpo" en Educación Física. Revista Iberoamericana de Educación, n.39, 25-51.

Behets, D. y Vergauwen, L. (2004). Value orientations of elementary and secondary physical education teachers in Flanders. Research Quarterly for Exercise and Sport, n.75, 156-164.

Bernstein, B. (1988). Clases, Códigos y Control. II. Hacia una Teoría de las Transmisiones Educativas. Madrid: Akal.

Bernstein, B. (1989). Clases, Códigos y Control. I. Estudios Teóricos para una Sociología del Lenguaje. Madrid: Akal.

Bernstein, B. (1993). La Estructura del Discurso Pedagógico. Madrid: Morata.

Biesta, G. (1998). Say you want a revolution... Suggestions for the impossible future of critical pedagogy. Educational Theory, n.48, 499-510.

Bourdieu, P. y Passeron, J. (1973). Los Estudiantes y la Cultura. (3ª Edición). Buenos Aires: Labor

Bunker, D. y Thorpe, R. (1982). A model for the teaching of games. Bulletin of Physical Education, n.18, 5-8.

Contreras, J. (1997). La autonomía del profesorado. Madrid: Morata.

Devís, J. y Peiró, C. (1992) (Eds.). Nuevas perspectivas curriculares en Educación Física: la salud y los juegos modificados. Barcelona: INDE.

Dyson, B. (2002). The implementation of cooperative learning in an elementary school physical education program. Journal of Teaching in Physical Education, n.22, 69-85.

Dyson, B.; Linehan, N. y Hastie, P. (2010). The ecology of cooperative learning in elementary physical education classes. Journal of Teaching in Physical Education, n.29, 113-130.

Elboj, C.; Puigdellívol, I.; Soler, M. y Valls, R. (2002). Comunidades de aprendizaje. Transformar la educación. Barcelona: Graó.

Ellsworth, E. (1989). Why doesn't this feel empowering? Working though the repressive myths of critical pedagogy. Harvard Educational Review, n.59, 297-323.

Ennis, C. (1997). Defining the dreaded curriculum: Tensions between the modern and the postmodern. En J. Fernández-Balboa (Ed.), Critical postmodernism in human movement, physical education, and sport (pp. 207-220). Albany, NY: State University of New York Press.

Evans, J.; Davies, B. y Rich, E. (2009). The body made flesh: embodied learning and the corporeal device. British Journal of Sociology of Education, n.30, 391-406. 
Fernández-Balboa, J. (1995). Reclaiming physical education in higher education through critical pedagogy. Quest, vol.47, n.1, 91-114.

Fernández-Balboa, J. (1997). Physical education teacher preparation in the postmodern era: Toward a critical pedagogy. En J. Fernández-Balboa (Ed.), Critical postmodernism in human movement, physical education, and sport (pp. 121-138). Albany, NY: State University of New York Press.

Foucault, M. (1988) The concern for truth. En L. Kritzman (Ed.), Michel Foucault. Politics, philosophy, culture: interviews and other writings 1977-1984. (pp. 255-267). London: Routledge.

Freire, P. (1978). Pedagogía del oprimido. Madrid: Siglo XXI.

Giddens, A. (1993). Consecuencias de la modernidad. Madrid: Alianza.

Giroux, H. (1990). Los profesores como intelectuales. Hacia una pedagogía crítica del aprendizaje. Barcelona: Paidós/MEC.

Goodson, I. (1988). The making of the curriculum, collected essays. Basingstoke: The Falmer Press.

Goodson, I. (1995). Historia del currículum. La construcción social de las disciplinas escolares. Barcelona: Pomares-Corredor.

Gore, J. (1993). The struggle of pedagogies: critical and feminist discourses as regimes of truth. New York \& London: Routledge.

Gore, J. (2003). What can we do for you! What can "we" do for "you"? Struggling over empowerment in critical and feminist pedagogy. In A. Darder, M. Baltodano \& R. D. Torrers (Eds.), The Critical Pedagogy Reader (pp. 331-348). New York: RoutledgeFalmer.

Griffin, L. y Butler, J. (2005). Teaching games for understanding. Theory, research, and practice. Champaign, IL: Human Kinetics.

Gur-Ze'ev, I. (1998). Toward a nonrepressive critical pedagogy. Educational Theory, vol.48, n.4, 431-463.

Hargreaves, A. (1996). Profesorado, cultura y postmodernidad. Cambian los tiempos, cambia el profesorado. Madrid: Morata.

Hellison, D. (2003). Teaching responsibility through physical activity. (2nd ed.). Champaign, IL: Human Kinetics.

Hellison, D.; Cutforth, N.; Kallusky, J.; Martinek, T.; Parker, M. y Stiehl, J. (2000). Youth development and physical activity. Champaign, IL: Human Kinetics.

Hellison, D. y Martinek, T. (2006). Social and personal responsibility programs. In D. Kirk, M. O'Sullivan, \& D. MacDonald (Eds.), Handbook of research in physical education (pp. 610626). London: Sage.

Hernández, J. (1996). La construcción histórica y social de la Educación Física: El currículo de la LOGSE, ¿una nueva definición de la Educación Física escolar? Revista de Educación, n.311, 51-76.

Hickey, C. (2001). "I feel enlightened now, but...": The limits to the pedagogic translation of critical social discourses in physical education. Journal of Teaching in Physical Education, vol.20, n.3, 227-246.

Hickey, C. (2005). Planificar el futuro, contra antecedentes poderosos y presentes precarios. En A. Sicilia y J. Fernández-Balboa (Eds.). La otra cara de la enseñanza. La educación física desde una perspectiva crítica (pp. 77-92). Barcelona: INDE.

Ibáñez, T. (2001). Municiones para disidentes. Realidad-Verdad-Política. Barcelona: Gedisa.

Jannosy, F. (1980). La fuerza del trabajo y el progreso ante los cambios permanentes de la calificación de los trabajadores. En G. Labarca (Comp.), Economía política de la educación (pp. 55-87). México: Nueva imagen.

Kirk, D. (1990). Educación física y curriculum. Valencia: Universidad de Valencia.

Kirk, D. (1993). Curriculum work in physical education: Beyond the objectives approach? Journal of Teaching in Physical Education, n.12, 244-265.

Lather, P. (1998). Critical pedagogy and its complicities: A praxis of stuck places. Educational Theory, n.48, 511-519. 
McKay, J.; Gore, J. y Kirk, D. (1990). Beyond the limits of technocratic physical education. Quest, n. $42,52-76$.

Nietzsche, F. (1996). Sobre verdad y mentira en sentido extramoral. ( $3^{\text {a }}$ Ed.). Madrid: Tecnos.

O’Sullivan, M.; Siedentop, D.,y Locke, L. (1992). Toward collegiality: Competing viewpoints among teacher educators. Quest, n.44, 266-280.

Ortega y Gasset, J. (2002). Misión de la universidad. Madrid: Alianza.

Pascual, C. (1999). La formación inicial del profesorado de Educación Física: En busca del significado profesional perdido. Conceptos de Educación, n.6, 75-90.

Pascual, C. (2006). The initial training of physical education teachers. In search of the lost meaning of professionalism. Physical Education \& Sport Pedagogy, n.11, 69-82.

Sánchez, M. (1999). Voices inside schools - La Verneda-Sant Martí: A school where people dare to dream. Harward Educational Review, n.69, 320-336.

Siedentop, D. (1994). Sport education: Quality PE through positive sport experiences. Champaign, IL: Human Kinetics.

Siedentop, D. (1998). What is sport education and how does it work? Journal of Physical Education, Recreation and Dance, vol.69, n.4, 18-20.

Siedentop, D. (2002). Sport education: A retrospective. Journal of Teaching in Physical Education, n.21, 409-418.

Sicilia, A. (2009). Economía, desigualdad y transformación social ¿Qué papel juega la educación física? En J. Moreno y D. González-Cutre (Eds.), Deporte, intervención y transformación social (pp. 211-241). Rio de Janeiro: Shape.

Sicilia, A.; Delgado, M.; Sáenz-López, P.; Manzano, J.; Varela, R.; Cañadas, J. y Gutiérrez, M. (2006). La evaluación de aprendizajes en educación física. Diferencias en función del nivel educativo. Motricidad. European Journal of Human Movement, n.17, 71-95

Sicilia, A. y Fernández-Balboa, J. (2005). La otra cara de la enseñanza. La educación física desde una perspectiva crítica. Barcelona: INDE.

Sicilia, A. y Fernández-Balboa, J. (2006) Ethics, politics and bio-pedagogy in physical education teacher education: easing the tension between the self and the group. Sport, Education and Society, n.11, 1-20.

Sicilia, A. y Fernández-Balboa, J. (2009). Reflecting on the moral bases of critical pedagogy in PETE: Toward a Foucaultian perspective on ethics and the care of the self. Sport, Education and Society, n.14, 443- 463.

Thorpe, R.; Bunker, D. y Almond, L. (1986) (Eds.). Rethinking games teaching. Loughborough:University of Technology.

Tinning, R. (1991). Teacher education pedagogy: Dominant discourses and the process of problem setting. Journal of Teaching in Physical Education, n.11, 1-20.

Tinning, R. (2002). Toward a "modest pedagogy": Reflections on the problematics of critical pedagogy. Quest, n.54, 224-240.

Velázquez, C. (2010) (Coord.). Aprendizaje cooperativo en Educación Física. Fundamentos y aplicaciones prácticas. Barcelona: INDE.

Woods, P. y Hammersley. M. (1995) (Comps). Género, cultura y etnia en la escuela. Informes etnográficos. Barcelona: Paidós/MEC.

Wright, J. (2000). Bodies, meanings and movement: A comparison of the language of a physical education lesson and a feldenkrais movement class. Sport, Education, \& Society, n.5, 35-49.

Wright, P. y Burton, S. (2008). Implementation and outcomes of a responsibility-based physical activity program integrated into an intact high school physical education class. Journal of Teaching in Physical Education, n.27, 138-154. 
